\title{
Dinámicas metropolitanas en la era de la globalización: la promoción inmobiliaria para empresas en la ciudad de São Paulo, Brasil
}

\begin{abstract}
We propose to discuss a distinguishing element of the metropolis in the present period: the real estate promotion for companies and their implications for the planning and the urban life, trying to consider the metropolis like a space that shelters a multiplicity of interests and conflicts. Our approach is centered in the city of São Paulo, host municipality of the greater metropolitan region of Brazil, doing to emphasize the characteristics that also share with other metropolis of peripheral countries and their specificities.
\end{abstract}

Key words: Metropolis, globalization, corporative real estate, São Paulo City

\section{Resumen}

Proponemos discutir un elemento distintivo de las metrópolis en el período actual: la promoción inmobiliaria para empresas y sus implicaciones para la planificación y la vida urbana, procurando considerar la metrópoli como un espacio que abriga una multiplicidad de intereses y de conflictos. Nuestro enfoque se centra en la ciudad de São Paulo, municipio sede de la mayor región metropolitana de Brasil, haciendo resaltar las características que comparte con otras metrópolis de países periféricos y también sus especificidades.

Palabras clave: Metrópolis, globalización, promoción inmobiliaria corporativa, São Paulo 


\section{Introducción}

$\mathrm{L}$ a metropolización, aunque sea un fenómeno mundial, no se muestra incólume a la influencia de cada formación socio-espacial (Santos, 1977) de la cual es parte, y a la cual ayuda a estructurar. De tal suerte, la especificidad de un país periférico imprime sus marcas y características a sus ciudades $y$, muy particularmente, a sus grandes metrópolis, tales como Caracas, Bombay, Ciudad de México, Río de Janeiro o São Paulo. Estas características dependen, entre otros factores, del grado de profundidad de la división social y territorial del trabajo, de la integración del territorio nacional, de sus redes internas de flujos materiales e inmateriales, de su papel en el proceso de globalización selectiva de la economía. Las especificidades locales y la manera como cada metrópoli se inserta en la economía globalizada, se hacen únicas. Se puede, no obstante, considerar que, en el período actual, las metrópolis de los países periféricos comparten algunas características y dinámicas que las diferencian de sus congéneres de los países centrales, aunque todas pertenezcan a lo que se podría identificar como una red mundial de metrópolis.

Proponemos discutir un elemento de estas características distintivas de las metrópolis de la periferia del sistema capitalista: la promoción inmobiliaria para empresas y sus implicaciones para la planificación y la vida urbana, procurando considerar la metrópoli como un espacio que abriga una multiplicidad de intereses y de conflictos.

Antes de adentrarnos en el debate principal de este artículo, se deben considerar dos puntos. El primero es que, como toda metrópoli, São Paulo es un lugar complejo, en el sentido que J-E Sánchez (1991) atribuye al término, y, como tal, está compuesto por una variedad de subespacios especializados y, añadiríamos, alcanzados y tratados de forma diferente por

* Geógrafa, Doctora en Geografía Humana por la Universidade de São Paulo, Profesora e Investigadora del Departamento de Geografía del Instituto de Geociencias de la Universidade Estadual de Campinas, Brasil. E- mail: abernar@ige.unicamp.br

** Geógrafo, Doctor en Geografía Humana por la Universidade de São Paulo, Profesor e Investigador del Departamento de Geografía del Instituto de Geociencias de la Universidade Estadual de Campinas, Brasil. E-mail: castillo@ige.unicamp.br. Enviado el 29 de septiembre de 2006, aprobado el 30 de marzo de 2007. las políticas públicas y por las inversiones privadas. Esto define diversas categorías de segregación urbana y, en el caso de las metrópolis de los países pobres, dirige las inversiones públicas hacia algunos sectores y cuadrantes de la ciudad.

El segundo punto sobre el que queremos llamar la atención es la magnitud y la enorme concentración de personas, factores y funciones que definen a las metrópolis de los países periféricos. De acuerdo con M. Santos (1994a, p. 19): "un elemento que tienen en común las metrópolis mundiales del Tercer Mundo procede /.../ del hecho de que, insertadas en una división internacional del trabajo que exige fluidez, la división territorial del trabajo propia de cada país padece limitaciones. Las restricciones existentes para una movilidad de los factores más completa, tienden a reforzar la posición de ciertas regiones y de ciertos lugares".

São Paulo es una ciudad crítica, donde la crisis se manifiesta incluso en función de las exigencias de las grandes firmas - al servicio de cuyos intereses la ciudad se estructura, atendiendo a sus demandas de fluidez y rapidez. Las nuevas necesidades de la producción exigen infraestructuras especializadas que respondan a los elevados niveles de competitividad requeridos por la economía globalizada. Los gobiernos municipales y de los Estados ${ }^{1}$ invierten para viabilizar el gran capital, y crean espacios funcionales dentro de sus perímetros administrativos que, en conjunto con las acciones de empresas privadas del sector inmobiliario, van dando un nuevo sesgo metropolitano a la ciudad, principalmente a partir de los años 1980. Es en este contexto, entonces, que la promoción inmobiliaria para empresas despunta como uno de los principales elementos de la actual función metropolitana de São Paulo.

\section{São Paulo: contornos de un nuevo período}

Un rápido bosquejo del perfil del municipio y de la Región Metropolitana de São Paulo (RMSP), a través del levantamiento de algunos elementos seleccionados, servirá como telón de fondo a los análisis que seguirán.

${ }^{1}$ Los Estados de la Federación Brasileña son una unidad político-administrativa equivalente a los Departamentos, Estados y Provincias en el ámbito hispanoamericano. 
El municipio de São Paulo es sede de 32 de las cien mayores empresas nacionales y 55 de las cien mayores empresas extranjeras instaladas en el país (Gazeta Mercantil, Balance Anual, 2004). Administra el tercer mayor presupuesto del país, precedido solamente por la Unión y por el Estado de São Paulo; posee 15,5\% de la flota nacional de vehículos -5,4 millones de vehículos, lo que da como resultado, aproximadamente, un vehículo por cada dos personas (Fundação Seade / Denatran, 2002)-. Los ómnibus, el metro y el sistema ferroviario de la periferia transportan millones de pasajeros por día.

Entre los treinta mayores bancos comerciales y múltiples en Brasil en 1999, por volumen de depósito, 63\% tienen sede en el municipio de São Paulo, 6\% en Río de Janeiro, 6\% en Brasília, 6\% en Porto Alegre y $6 \%$ en Salvador (Fundação Seade, 2000).

Estos datos revelan que hay una fuerte concentración del sistema financiero nacional en São Paulo y en su región metropolitana, en detrimento de las antiguas plazas financieras regionales que, poco a poco, se fueron volviendo tributarias del gran conglomerado de la capital paulista (Corrêa, 1989). Este hecho deja inevitablemente sus huellas en el paisaje de la ciudad: ha generado grandes alamedas de bancos en áreas muy valorizadas, que presentan una arquitectura vanguardista, como son los casos de la Avenida Paulista, de la Avenida Faria Lima, de la Avenida Luiz Carlos Berrini y, hoy por hoy, de la Avenida de las Naciones Unidas, y que demandan siempre nuevas áreas especialmente proyectadas. Retornaremos a este punto.

La concentración del tráfico aéreo en São Paulo, por su parte, también demuestra su papel como conexión privilegiada de inmensos flujos de mercancías y personas en relación con todo el territorio brasilero. En cuanto a los flujos aéreos en Brasil en 2005, la RMSP, en sus tres principales aeropuertos, concentró $34,5 \%$ de los pasajeros, 37,8\% de la carga y $21,8 \%$ de la valija postal (Infraero, 2006).

Las estadísticas nos indican, no obstante, que hay una pérdida de la centralidad de la RMSP en lo que atañe a las nuevas inversiones industriales, y que, incluso así, permanece en la metrópoli la centralidad de la toma de decisiones, de control y de comando (Lencione, 2003). En 1970, la RMSP respondía por $44 \%$ del Valor de la Transformación Industrial
(VTI) nacional; mientras que en 1980 por 33\% del VTI nacional. Por lo tanto, los años 1970 marcan el inicio del proceso de reversión de la concentración industrial, acompañada de una desaceleración del crecimiento del sector en la RMSP. Entre los principales motivos de la desconcentración industrial en la RMSP, pueden mencionarse los siguientes: tráfico congestionado; salarios elevados en el área central; precio de la tierra y tributos fiscales elevados; restricciones legales y ambientales; intensidad de la acción sindical; intensificación de la guerra fiscal entre Estados y entre municipios y otros fenómenos de la competencia entre los lugares (Emplasa, 1994, p. 37).

La desconcentración industrial selectiva ${ }^{2}$ está acompañada por una concentración de los servicios superiores o cuaternarios, es decir, servicios al sector de los servicios, en la ciudad de São Paulo. Las nuevas actividades productoras de información, agrupadas en el sector cuaternario de la economía, están centradas en la elaboración y coordinación de las múltiples redes planetarias. Según M. E. Hepworth (1989), los trabajos con la información definen cambios en la estructura productiva y de transacciones de las economías contemporáneas. Son sectores que engloban novísimas empresas y profesiones, que vienen a impulsar la actual división territorial del trabajo y, por lo tanto, las nuevas dinámicas urbano-regionales (Marshall, 1994) alrededor del mundo. Como han afirmado J. Weeler y R.L. Mitchelson (1989, p. 540) la génesis de la información es fuertemente jerárquica, y un rico campo de informaciones financieras, jurídicas, entre otras, está concentrado en pocos lugares.

Así, la nueva centralidad de la información en São Paulo garantiza la presencia de actividades sofisticadas, tales como, finanzas, informática, consultorías y otras funciones administrativas especializadas, publicidad y marketing, que acompañan a las transformaciones cualitativas de la industria en busca de la reestructuración y, por consiguiente, de mayor competitividad. Las diez mayores agencias de publicidad del mundo actúan en Brasil y todas se localizan en el municipio de São Paulo (Ibope Mo-

${ }^{2}$ La desconcentración selectiva ocurre en doble sentido, una vez que, no sólo es seleccionado el lugar hacia donde migra la unidad productiva, sino también el tipo o ramo de la industria que sale. 
nitor, 2005). Entre las mega-agencias globales se destacan: Young \& Rubican, Publicis Salles Norton, Ogilvy Brasil, J. Walter Thompson, McCann Erickson, Lew Lara, Almap BBDO, DM9DDB, F Nazca y Giovani FCB. La inversión en el mercado publicitario en Brasil casi se duplicó entre 1997 y 2003, pasando de 6.800 millones de reales a 12.000 millones de reales (Mídia São Paulo, 2004; Meio \& Mensagem, 2004).

São Paulo acoge las oficinas de las grandes empresas transnacionales de consultoría que actúan en el territorio brasilero. Entre estas empresas se destaca el grupo conocido como Big Five, que domina gran parte del mercado brasilero y latinoamericano de consultoría y auditoría: Price Waterhouse Coopers, Andersen Consulting (ahora Accenture), KPMG, Ernst \& Young y Delloitte Touche. Además de las empresas del Big Five, sobresalen también otras empresas de consultoría tales como McKinsey \& $\mathrm{CO}$, Consulting, Mercer Consulting Groupe, BoozAllen \& Hamilton. Estos datos indican que la ciudad de São Paulo retiene una colosal densidad de información, y por lo tanto ejerce un importante papel, sobre este aspecto, como centro de gestión y principal conexión, en el país, de las redes globales de la información corporativa (Silva, 2004). Es importante enfatizar, por consiguiente, que son las empresas productoras de informaciones las que impulsan las transformaciones sustanciales en la división del trabajo contemporánea, transformando las metrópolis donde se concentran en verdaderos "complejos corporativos" (Marshall, 1994; Wood, 1996; Goe, 1996).

El rol de São Paulo como eje o médula de los servicios superiores o cuaternarios le confiere, actualmente, la condición de región privilegiada en cuanto a las inversiones del sector inmobiliario de oficinas. La acelerada transformación y paso de la manufactura a los servicios en algunas ciudades implicó el desvío de grandes inversiones en capital fijo de la industria hacia sofisticadas oficinas (Beauregard, 1991). En lo que concierne a su ubicación en el propio municipio, los servicios más sofisticados mantuvieron una evolución en torno al eje centro-sudoeste. En la década de 1960, los alrededores de la Praça da Sé (área central de la ciudad) constituían el lugar privilegiado. A ello le siguió el traslado hacia la Avenida Paulista en la década de 1970, donde se nota ya una preocupación más intensa con la infraestructura especializada y con una arquitectura de estilo vanguardista, si bien aún se puede notar un uso mixto del área, debido a la permanencia en el lugar de algunos edificios residenciales. Las décadas de 1980 y 1990 testimonian el surgimiento grandioso de lo que se podría denominar como producción inmobiliaria para empresas propiamente dicha, esto es, los ejes que abarcan las avenidas Faria Lima, Luis Carlos Berrini y Marginal Pinheiros (Avenida Naciones Unidas), con su infraestructura especializada (telefonía, telemática, sistema vial accesible, aparcamiento), leyes de uso del terreno y otras normas que favorecen el uso corporativo de estas áreas de la ciudad.

\section{La promoción inmobiliaria para empresas y las metrópolis contemporáneas}

Un conjunto de inmuebles destinados a grandes empresas, planeados y proyectados como centro de negocios, generalmente por firmas especializadas en este sector de la construcción y promoción inmobiliaria, ya sea valiéndose de una arquitectura atrevida y de estilo vanguardista o con un tratamiento paisajístico meticuloso: he aquí una descripción de lo que los franceses llaman immobilier d'entreprise. Según Malézieux (1996, p. 42), "la realidad que designa el término immobilier d'entreprise no es nueva, puesto que consiste en el conjunto, de edificios y espacio, construido y planeado para acoger un establecimiento de una empresa secundaria o terciaria, para el ejercicio de una o varias funciones relacionadas con la producción de bienes y de servicios". La promoción inmobiliaria para empresas gana autonomía en Europa, constituyéndose en un ramo particular de producción; en otras palabras, surgen firmas especializadas en la concepción, la producción, el arrendamiento y la administración de inmuebles para empresas (Castillo, 2000).

Una de las consecuencias más acentuadas de este proceso son las estrategias de valorización agraria e inmobiliaria, que son resultado de una convergencia entre la propiedad agraria, la inversión en el sector y el prematuro carácter obsoleto de las áreas aledañas a las inversiones, con la expulsión frecuente de los habitantes considerados escasamente acordes con la nueva situación. 
Uno de los grandes motores de este tipo de promoción inmobiliaria es la denominada guerra de los lugares (Santos, 1994b y 1996), esto es, una competencia desenfrenada entre las ciudades para atraer empresas e inversiones - recurso muy aplicado en tiempos de crisis económica, y del que se pueden encontrar innumerables ejemplos en Europa, Estados Unidos y Brasil.

De ahí el porqué de que las municipalidades, sustentadas por una institucionalidad que les garantiza cierta autonomía fiscal y política, se empeñen en hacer acuerdos con empresas del ramo inmobiliario para promover atrevidos centros empresariales dentro de sus circunscripciones. Se trata de los consorcios de crecimiento de que habla M. Gottdiener (1993). En algunas regiones metropolitanas, verdaderos mosaicos de poderes públicos locales, la competencia entre las diversas municipalidades conduce a una superoferta de este tipo de mercancía, inmovilizando capital en construcciones ociosas. En París, ciudad que presentaba a mediados de los años 1990 el mayor parque de oficinas en metros cuadrados de Europa, muchos centros empresariales nuncallegaron a ser ocupados ${ }^{3}$.

Según P. Delsaut (1992, p. 23), el aumento de la movilidad de las empresas ante una competencia internacional cada vez más reñida, contribuye a la irrupción de la promoción inmobiliaria para empresas en determinadas regiones desde, al menos, los años ochenta.

La lógica de la destrucción y reconstrucción característica del circuito inmobiliario (Lefebvre, 1976; Harvey, 1980; Gottdiener, 1993) reside, de alguna forma, en la pugna entre la búsqueda de una racionalidad utilitarista y los conflictos originados por las inmensas carencias de infraestructuras y servicios públicos. Los lugares tratan de atraer inversiones públicas y privadas, por medio de intervenciones materiales y normativas, generando subespacios corporativos y, con frecuencia, saqueando el erario pú-

${ }^{3}$ En 1992, el parque de oficinas de París contaba con cerca de 40 millones de $\mathrm{m}^{2}$, siguiéndoles los parques de oficinas de Londres, con cerca de 35 millones de $\mathrm{m}^{2}$, Frankfurt, Milán y Berlín, con alrededor de 10 millones de $\mathrm{m}^{2}$, y Madrid con 5 millones de $\mathrm{m}^{2}$ (Delsaut, 1992, p. 38). blico; las empresas, por su parte, buscan los lugares más adecuados para cada una de sus actividades. En algunos casos, particularmente en las metrópolis, este fenómeno crea las condiciones para la intervención de firmas globales especializadas en levantar y administrar inmuebles de oficinas de altísimo nivel. Los consorcios para el crecimiento, por lo tanto, en lo que atañe a la producción de este nuevo espacio hegemónico, tienden, cada vez más, a depender de la dinámica de la economía globalizada.

La producción de estos espacios de la globalización implica, tanto en lo que respecta a su forma, como a su función, la constitución de un poderoso circuito inmobiliario fuertemente transnacionalizado. Con ello, toda una organización, un nuevo ritmo temporal, se impone al espacio de una metrópoli de país periférico. Se crea una concepción racionalizada del espacio, los llamados espacios de la racionalidad (Santos, 1994 b y 1995).

\section{Particularidades de la ciudad de São Paulo}

Todas las acciones que se ejecutan sobre el territorio se hacen con base en un conjunto de formas y de reglas en sistema, que causan constantes desorganizaciones y reorganizaciones del espacio con diversos niveles de intensidad, y que impiden, por consiguiente, que un subsistema hegemónico dado exista cerrado en sí mismo.

El uso corporativo y racional del territorio nunca se da de forma plena, una vez que encuentra y se combina con un conjunto heterogéneo de formas y usos, técnicas y normas no funcionales a las acciones hegemónicas, y que se pueden convertir en obstáculos, resistencias, finalidades contraproducentes.

La región sudoeste ${ }^{4}$ de la ciudad de São Paulo, a la que dirigimos nuestro análisis, ha vivido en las últimas décadas un acelerado proceso de transformación que altera sus antiguas funciones resi-

\footnotetext{
${ }^{4}$ Por región sudoeste estamos considerando lo que $\mathrm{H}$. K. Cordeiro (1980) denomina "cuadrante sudoeste, un área formada por los barrios de Jardins, Brooklin, Vila Olímpia, Itaim, Santo Amaro (Chácara Santo Antonio) y Alto de Pinheiros, que tienen como ejes principales de circulación vial, la Avenida Paulista, Avenida Faria Lima, Avenida Berrini y Marginal Pinheiros".
} 
Figura 1. Ejes principales del nuevo centro corporativo de la Ciudad de São Paulo

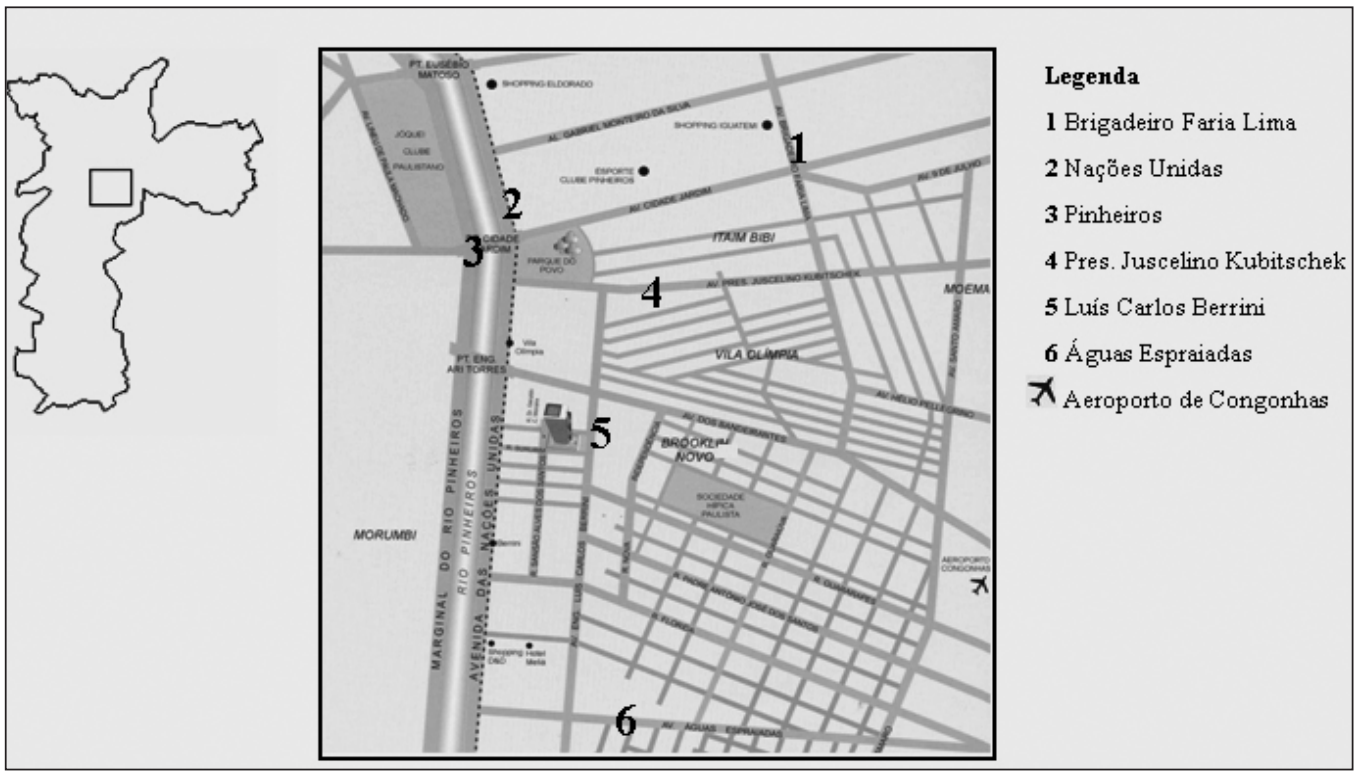

Fuente. Prefeitura Municipal de São Paulo

denciales. Desde mediados de la década del 70, y sobre todo a partir de la década del 90, este espacio de la ciudad viene siendo ocupado por sofisticados edificios de oficinas que acogen las sedes de grandes empresas industriales, empresas de servicios de alto nivel y algunos ramos del sector financiero (Figura 1).

H. K. Cordeiro (1980), en su clásico estudio sobre la expansión del centro de negocios de la metrópoli paulista, nos ayuda a encontrar la génesis de este proceso de construcción de inmuebles destinados a empresas que se inicia en la década del 70 en São Paulo con el crecimiento del antiguo centro metropolitano hacia la Avenida Paulista y luego hacia la Avenida Faria Lima. Según la autora, "el cuadrante sudoeste se consume cada vez más por la cotidiana expansión del Centro Metropolitano, donde se instalan los servicios terciarios y cuaternarios." Las zonas residenciales fueron ocupadas rápidamente por estas nuevas actividades, "así como por las vías de rápida circulación, y al fin, la ley de división por zonas vino a oficializar una situación irremediable." (Cordeiro, 1980, p. 73).
Pero, son dos eventos en particular los que marcan el punto de partida de la promoción inmobiliaria para empresas en São Paulo: la creación del Centro Empresarial São Paulo y de la Avenida Luiz Carlos Berrini, construidos a finales de la década del 70 . Por primera vez en la historia urbana de São Paulo se emprendieron, estratégicamente y a una misma vez, dos grandes proyectos inmobiliarios dedicados exclusivamente a servir de sede a las actividades de gestión. Estos proyectos dieron inicio a un nuevo momento de especulación inmobiliaria en la ciudad, relacionado directamente con la formación de un espacio de globalización paulista que, de ese modo, garantizaba las nuevas formas globalizadas de circulación del capital inmobiliario.

El Centro Empresarial São Paulo y los edificios de la Avenida Berrini tienen en común, además de las funciones, su ubicación estratégica: cerca de la avenida Marginal Pinheiros, con extensas áreas disponibles para mega proyectos a precios competitivos, bien atendida por vías expresas, acceso fácil al aeropuerto de Congonhas, división en zonas favorable y demás infraestructuras suministradas por el poder público (Gaeta, 1995 y Fujimoto, 1994). 
El Centro Empresarial São Paulo, condominio cerrado y autosuficiente, abriga 45 empresas, 90 tiendas, 8 agencias bancarias, 30 restaurantes, garaje para 5.400 coches en seis torres y un centro comercial por donde circulan cerca de 15.000 personas cada día (www.centroempresarial.com.br, 2006). En él se encuentran oficinas sedes de corporaciones tales como Bombardier, Procter\&Gamble, Alcoa, Rhodia, Unilever, Bunge, entre otras. El proyecto de la obra fue realizado por Lubeca, brazo inmobiliario del grupo Moinho Santista (Gaeta, 1995).

A su vez, la Avenida Berrini tiene la particularidad de haber sido rediseñada en sus funciones de una sola vez y por un único grupo inmobiliario: la empresa Bratke-Collet. Hasta el final de la década de los 80 , el $95 \%$ de los 24 edificios construidos en la avenida pertenecía a Bratke-Collet, lo que muestra el carácter monopolista de esta firma. A mediados de los años 70 la empresa recibió los capitales necesarios rápidamente, y ya a finales de esa década, en aproximadamente dos años, había entregado el primer lote de edificios a los compradores, la mayoría instituciones financieras privadas y propietarios rentistas.

El grupo Bratke-Collet es un ejemplo del carácter monopolista y autónomo del sector inmobiliario en São Paulo, así como del carácter flexible de su actuación. El corto plazo de construcción de los edificios fue posible, fundamentalmente, gracias al conocimiento tecnológico adquirido en el sector de la ingeniería civil. Esto, por una parte, y por otra, debido a la utilización de recursos como el de la subcontratación, tanto en la construcción como en la administración de empresas. Así, se contrataron empresas de ingeniería especializadas en hidráulica, electricidad y cimientos. La mano de obra no calificada también fue contratada a terceros (Fujimoto, 1994).

Aceleradamente, se edifica en una parte de la ciudad un nuevo centro de negocios corporativos. En 1970, de las mayores empresas con oficinas en la capital paulista, el 54\% se encontraban en la región del Centro Antiguo, 14\% en la Avenida Paulista y $11 \%$ en la región de Marginal Pinheiros. Para confirmar el cambio de la centralidad de la capital, los datos de 1998 muestran que el 41\% de las mayores empresas tenían sus oficinas en la región de la Marginal, $21 \%$ en la Paulista y solo el $18 \%$ en la región del Centro Antiguo (Folha de S. Paulo, 30/11/2003; CB Richard Ellis, 2005).

Así, en las zonas aledañas a la Marginal Pinheiros y a la Avenida Berrini, principalmente en los barrios de Itaim, Vila Olímpia y Brooklin crecen, año tras año, las inversiones inmobiliarias para las empresas. Hay un fuerte dinamismo, con cierta tendencia a la expansión, para este sector de inversiones inmobiliarias en São Paulo. Hacia esta región se mudaron, en la década del 90, el Lloyds Bank, el Banco Noroeste, el Swiss Bank, el Banco Marco, la AT\&T. Son innumerables los ejemplos de reubicación de las sedes de las empresas en dirección al sector sudoeste de la ciudad, provenientes tanto de la propia región metropolitana como de otras ciudades del territorio brasileño. Según informaciones de la Bolsa de Inmuebles de São Paulo (BIESP), que también asesora inversiones en esta región de la ciudad, la tasa de desocupación de estos inmuebles, en el 2005, se mantuvo alrededor del 15\%. Además, esta es la región que más absorbe las nuevas reservas de oficinas de la ciudad: $65.000 \mathrm{~m}^{2}$ de los $115.300 \mathrm{~m}^{2}$ ofertados en 2005.

A pesar de todo, la edificación del nuevo centro de negocios en la ciudad de São Paulo ocurre en medio de luchas entre distintos intereses corporativos. De un lado, se encuentra la coalición que respalda los proyectos en la región sudoeste de la ciudad, produciendo un complejo medio técnico científico y de información (M. Santos, 1994) en la metrópoli paulista con el apoyo del poder local. De otro lado, se encuentra la reciente alianza polarizada en torno a un proyecto de revitalización del centro histórico de la ciudad, la cual concentra todavía a cerca del $50 \%$ del sector financiero de una urbe que se define también por ser la primera plaza financiera del país. Esta última coalición, a través de la Asociación Viva el Centro, que preside el Banco de Boston, tiene como objetivo realizar una profunda revitalización del antiguo centro comercial y de negocios, convirtiéndolo, según el modelo de las metrópoli europeas, en un lugar de negocios transnacionales, dotado con un complejo turístico y áreas de ocio y consumo sofisticados. Ha sido práctica corriente en las negociaciones, con el fin de legitimar la coalición Viva el Centro, la conformación de un discurso sobre las ventajas que São Paulo tendría como ciudad global, hecho este solamente posible, según afirman, cuan- 
do su centro histórico posea "una identidad que lo destaque y valorice entre las demás ciudades”: São Paulo sería entonces "una ciudad global y capital del Mercosur (Informe Viva o Centro, no 73, mayo, 1996). Como resultado, el centro histórico de São Paulo es escenario de conflictos diarios entre las fuerzas que buscan la "revitalización" o "ennoblecimiento" y los sujetos responsables por todo tipo de actividades no hegemónicas -merolicos, pequeñas empresas artesanales, bares y restaurantes de cuarta categoría, etc. Estos últimos han sido tratados como fuente de problemas de seguridad e higiene públicas ${ }^{5}$.

Las instituciones financieras privadas poseen el control de la reorganización de la metrópoli. Son esas instituciones las que presiden las asociaciones responsables por las directrices e intervenciones urbanas relativas a los centros de negocio de la ciudad: el Bank Boston está entre los fundadores de la Asociación Viva o Centro; el Banco Itaú coordinó el movimiento Paulista Viva; el Unibanco, a su vez, se encargó del proyecto Aguas Espraiadas (Frúgoli Jr, 2000 y Silva, 2003).

Por lo general, los políticos, los medios de comunicación y los inversionistas, justifican esta nueva expansión del centro, alegando que las antiguas áreas -el Centro Histórico de la ciudad y la Avenida Paulista - están saturadas y son obsoletas tecnológicamente. En realidad, se trata de un proceso pautado en una renovada forma de especulación inmobiliaria que esconde intereses de instituciones financieras, intereses de la elite local y de sectores del mercado inmobiliario nacional y transnacional, entre otros agentes. Pero, de manera especial, se trata de un proceso que envuelve las estrategias arrendatarias de las corporaciones globales, ávidas de un medio metropolitano que les permita proyectar acciones más competitivas (Veltz, 1997).

La reorganización vial del cuadrante sudoeste caracteriza muy bien el poder de estas coaliciones. Incluso frente a la resistencia de varias instancias, fue creado un gran paso vial, para hacer fluir el tránsito, que conecta en poco tiempo los barrios de Pinheiros y Brooklin, así como fue canalizado el arroyo por

${ }^{5}$ Para comprender los pactos que hicieron posible el cambio del centro en la ciudad de São Paulo, consultar, entre otros, H. Frúgoli Jr (2000); A.F.A. Carlos (2001); M. Fix (2001). donde ahora pasa la Avenida Águas Espraiadas ${ }^{6}$, junto a la avenida Berrini, y se construyó un anillo vial que conecta la Marginal Pinheiros a la Avenida Berrini. Se ha concluido también la construcción de dos túneles denominados inteligentes, pues son monitorizados las 24 horas del día, a través de un circuito cerrado de televisión de la Compañía de Ingeniería del Tránsito, la misma empresa que instaló un control digitalizado del tráfico (semáforos inteligentes) en la región.

Como vemos, el Estado continúa siendo el gran mediador en el creciente proceso de hacer de la ciudad un ente de carácter corporativo. Reorganizar la estructura espacial pretérita, implantando macroobjetos técnicos con el fin de regular la fluidez y permitir la supervaloración del terreno, significar crear un nuevo subespacio en la metrópoli que garantice las condiciones de competitividad para grandes corporaciones. Ahora bien, esto trae consigo voluminosos recursos selectivamente gastados, en detrimento de una población carente de infraestructura y de equipamientos básicos. Así, el orden público reproduce y hace viable los intereses del orden corporativo, generando un proceso de supervaloración de determinadas áreas de la ciudad, reforzando, también, las condiciones de São Paulo como espacio de globalización.

Una gran parte de las inversiones de capital para la contratación de la construcción de los edificios en la región sudoeste, proviene en la actualidad de los denominados "fondos de pensión" o de "jubilaciones". En Brasil estos fondos, que crecen con la privatización de los beneficios de la seguridad social, invirtieron, en los años 90 , cerca del $15 \%$ de sus activos en inmuebles, que, en muchos casos, estaban destinados a empresas. Treinta fondos de pensión

${ }^{6}$ Donde ahora existe esta avenida, residían $3.220 \mathrm{fa}$ milias en el llamado Complejo de Favelas de Aguas Espraiadas. La OAS, constructora encargada de una gran parte de la obra, negoció la retirada con los residentes, que ahora viven en la periferia este de la ciudad, a $25 \mathrm{Km}$ del centro donde trabajan (Fujimoto, 1994). Vale subrayar que la periferia metropolitana no está provista de los servicios públicos esenciales como hospitales, escuelas, distracción, transporte, etc. Estos se localizan, mayoritariamente, en el centro extendido de la ciudad. Informes bien detallados, así como un análisis cuidadoso sobre la desocupación del área donde se localizaba el Complejo de Favelas de Águas Espraiadas pueden ser consultados en el trabajo de M. Fix (2001). 
fueron los responsables por el $85 \%$ de las cuotas del World Trade Center de São Paulo y el fondo de pensión de la Philips (PPS) posee uno de los mayores contratos de arrendamiento inmobiliario del país: la oficina central de Nestlé en Brasil. Entre 1990 y 1998, el total de las inversiones de esos fondos en el mercado inmobiliario se cuadruplicó, al aumentar de 2 a 8 miles de millones de dólares. En esta década, los principales fondos de pensión que invirtieron en la región sudoeste de la ciudad de São Paulo fueron Funcef, Previ, Petros y Sistel.

Otro súper proyecto, el Centro Empresarial Naciones Unidas, conjunto de tres torres de oficinas calificadas de inteligentes (una de ellas con 160 metros de altura), nos parece también un magnífico ejemplo para representar el comportamiento del actual período. Se trata de una asociación entre el fondo de pensión de la Caja Económica Federal (Caixa Econômica Federal) -organización que recibió el contrato-, la Método Engenharia y Tishman Speyer Properties. Esta última, una empresa del sector inmobiliario norteamericano, actuó por primera vez en América Latina. La función de esta empresa, en joint-venture con la Método Engenharia, era la de elaborar el proyecto, concebir las especificaciones técnicas, comercializar y administrar las ganancias (Gazeta Mercantil, 28/06/1996).

En los últimos años, varios "edificios inteligentes" despuntaron en el paisaje de la ciudad como signos de la globalización, dando forma a un paisaje postmoderno en la región sudoeste de la ciudad. Esta nueva disposición de objetos hace que São Paulo adquiera cierta semejanza física con las denominadas global cities (Sassen, 1991). Entre 1996 y 2006 fueron edificadas en São Paulo 22 torres para oficinas clasificadas de alto nivel (AA); de este total de edificios, 20 se localizan en el nuevo centro corporativo, en los alrededores de la Avenida Naciones Unidas.

Estos nuevos edificios nacen conectados a las redes globales y conforman, por consiguiente, la unicidad técnica planetaria (Santos, 1996 y 2000).

\footnotetext{
${ }^{7}$ Los nuevos inmuebles corporativos se construyen con alta tecnología, se diseñan para adaptarse constantemente a las innovaciones venidas de la telemática. De ahí los atributos de flexibilidad y de inteligencia para designar esos objetos.
}

La producción de inmuebles para grandes empresas tiende a ser normalizada globalmente, como nos indican los proyectos elaborados por la organización Council on Tall Buildings and Urban Habitat, especializada en asesorar y normalizar la edificación de tales inmuebles. Después del dossier para Europa y Asia, fue preparado el estudio titulado "Edificios Inteligentes Latinoamericanos". Se trata de una plataforma técnica y normativa unificada, que conduce a la estandarización de objetos y procedimientos, para producir mejor un ritmo de negocios a escala planetaria. De aquí que sea posible, en el período actual, una división del trabajo planificada, esto es, anticipada. En estos ambientes, todo es pensado y regulado para que funcione de una manera sistémica, en el menor tiempo y del modo más racional que sea posible. Por eso los actuales inmuebles destinados a empresas constituyen objetos técnicos informatizados, tanto en lo que concierne a la materialidad, pues conllevan un enorme volumen de información para ser construidos, como en lo relacionado a las funciones, o sea, desarrollar, disponer y distribuir información, poniendo en movimiento el sistema de acciones de las corporaciones.

Hay, fundamentalmente ahora, una nueva economía urbana local asociada en gran parte a la globalización, cuya consecuencia es una "nueva economía política en la ciudad de São Paulo" (Santos, 1994a). Además de aquellos sectores relacionados con el financiamiento, la contratación de las obras y la construcción de estos modernos inmuebles destinados a empresas, sobresale un enorme complejo de actividades que incluye a arquitectos, economistas, corredores, abogados, consultores, analistas de sistemas, agencias de publicidad y marketing especializadas. Se conforma todo un nuevo mercado de servicios que ofrece soporte a este circuito inmobiliario fuertemente globalizado. Empresas de consultoría inmobiliaria -como las grandes norteamericanas, Mackenzie Hill y Richard Ellis, radicadas en Brasil desde los años 1970 - no sólo asesoran a las empresas que reciben los contratos o a los propietarios, sino también a los inquilinos que buscan aquellos lugares mejor capacitados en tecnologías de la información. El centro de negocios paulista tiende, pues, a convertirse en una forma urbana bajo el mando de un "modelo universal", a través de una unicidad técnica y normativa planetaria y, por consiguiente, extremadamente rígida. 


\section{Consideraciones finales: tensión entre el orden global y el orden local en la actual formación metropolitana}

El peso del conglomerado paulista en relación al territorio nacional se ha consolidado a causa de la relativa escasez de recursos en la producción de infraestructuras. A lo largo del siglo XX el territorio brasileño se fue equipando desigualmente a favor de São Paulo. Además, la modernización espacialmente selectiva que tiene lugar en la metrópoli excluye crecientes grupos de población del uso de infraestructuras instaladas en la ciudad.

Es necesario, además, hacer énfasis en que el lugar que acoge a la economía cuaternaria exige la fluidez del territorio, una vez que se impone una economía de flujos. La fluidez propiciada por la globalización tiene relación con el fortalecimiento del poder de dirección del mercado mundial, favoreciendo las acciones de las grandes empresas que siguen la tendencia de la separación entre unidades de producción y unidades administrativas; estas últimas se concentran en las grandes ciudades. Sin embargo, permanecen en la metrópoli las pequeñas empresas no modernas que, de manera general, predominan en áreas al margen de los flujos hegemónicos y son resultado de las organizaciones pretéritas del espacio. La relación contradictoria que se establece, manifiesta la particularidad de una metrópoli que, al volverse rígida, acogiendo tiempos hegemónicos, encuentra, en los tiempos más lentos del orden local, sus verdaderas oportunidades de flexibilidad. El problema que debe ser señalado, en el caso brasilero, es que las actuales "coaliciones de crecimiento" (Gottdiener, 1993), al obedecer, nuevamente, a las necesidades de las grandes corporaciones, canalizan recursos para intereses ajenos, foráneos, en detrimento de las demandas locales de una población extremadamente menesterosa. De ahí la inexistencia, por ejemplo, de un proyecto social que se ocupe de esas pequeñas empresas.

Son justamente estas temporalidades diferentes, aunque convergentes, de las cuales nos habla $\mathrm{M}$. Santos (1994a), las que permiten comprender la producción de espacios especializados y selectivos en la metrópoli. Los tiempos hegemónicos y los tiempos hegemonizados (a cada uno de los cuales corresponde un conjunto de objetos propios y diferentemente fechados, ubicados en la ciudad) combinados, nos brindan el movimiento de la totalidad del lugar metropolitano. "Cada vez que la ciudad moderniza una de sus partes, ella, concomitantemente, decreta el envejecimiento prematuro de las demás áreas. Esto tiene consecuencias para la economía urbana, pues su renovación casi siempre constituye una respuesta a las necesidades de empresas más modernas" (Santos, 1994a, p. 99). Es bueno recordar constantemente que la renovación urbana que precede o que sigue a grandes inversiones privadas en promoción inmobiliaria para empresas, se financia con el dinero público, pero sus beneficios se reparten de manera desigual. Así, los procesos de promoción inmobiliaria para empresas que tienen lugar en São Paulo se basan en una lógica de modernización selectiva y de mercado segmentado. La competencia a escala global, de este modo, hace aún más desigual la apropiación de los espacios seleccionados de la ciudad.

Es fundamental, por tanto, tener en cuenta el poder de influencia de las grandes firmas en la estructuración de la metrópoli, en particular, de las firmas que reciben contratos inmobiliarios, sector clave de la producción del espacio urbano, como nos recuerdan H. Lefebvre (1976), M. Gottdiener (1991) y D. Harvey (1980).

Se constatan de esta manera enormes desigualdades internas en la aglomeración urbana paulista. De los 10,6 millones de habitantes del municipio de São Paulo, la gran mayoría encuentra algún tipo de dificultad de acceso a la salud, la educación, el hogar, o la renta. El número de habitantes de las favelas alcanza casi 1,2 millones, aglomerados en 279.070 domicilios en 2.018 favelas; el contingente de habitantes de los barrios bajos sobrepasa los 900 mil y los que viven en las calles llegan a las 8.200 personas (PMSP/FIBGE, 2000).

El déficit habitacional en la Región Metropolitana de São Paulo, a su vez, es de 596 mil viviendas (Ministério das Cidades, 2004) y, de acuerdo con el Centro de Estudios de la Metrópoli (2003), cerca de 7,2 millones de personas en RMSP viven en la línea de pobreza.

Este sería un primer cuadro de una "metrópoli corporativa y fragmentada” (Santos, 1990): por un 
lado, niega a la mayoría de sus habitantes al acceso a sus equipos más modernos; por otro, se constituye en una ciudad omnipresente en todo el territorio brasileño, que cumple un papel fundamental en la inserción de Brasil en el mundo globalizado.

Dentro de esta dinámica que combina las formas y procesos más disparatados y contradictorios, se puede visualizar la ciudad de São Paulo como importante conexión de la economía de flujos en el territorio nacional, eslabón de la economía mundial y asilo para un inmenso contingente de pobres urbanos. En este contexto, la demanda por la renovación urbana y la promoción inmobiliaria específicamente dirigida a las corporaciones, exacerba las tensiones y los conflictos.

\section{Referencias Bibliográficas}

Beauregard, R. A. (1991). "Capital restructuring and the new built environment of global cities: New York and Los Angeles". International Journal of Urban and Regional Research, 15, 1, 90-105.

Benko, G. (1990). La dynamique spatiale de l'économie contemporaine. Paris: Éditions de L'Espace Européen.

Bonamy, J. \& May, N. (1994). Services et mutations urbaines. Paris: Anthropos.

Carlos, A. F. A. (2001). Espaço-tempo na metrópole. São Paulo: Contexto.

Castillo, R. (2000). Aspects de la dynamique du secteur immobilier à São Paulo: du lotissement a l'immobilier d'entreprise. En: L. C. Dias et al. (orgs.), Villes et régions au Brésil. Paris: L'Harmattan.

Cordeiro, H. K. (1980). O centro da metrópole paulista. Expansão recente. São Paulo: Instituto de Geografia, Universidade de São Paulo

Corrêa, R. L. (1989). "Concentração bancária e os centros de gestão do território". Revista Brasileira de Geografia, 30, 2, 17-32.

Daniels, P. (1993). Service industries in the world economy. Oxford/Cambridge: Blackwell.

Delsaut, P. (1992). "L'immobilier d'entreprise et l'Europe". Cahiers de l'IAURIF, 100.

Emplasa. (1994). Plano Metropolitano da Grande São Paulo. São Paulo: Emplasa.

Fix, M. (2001). Parceiros da exclusão: duas historias da construção de uma nova cidade em São Paulo:
Faria Lima e Águas Espraiadas. São Paulo: Editora Boi Tempo.

Frúgoli J. H. (2000). Centralidade em São Paulo. Trajetórias, conflitos e negociaçôes na metrópole. São Paulo: Cortez / Edusp.

Fujimoto, N. (1994). A Produção Monopolista do Espaço Urbano e a Desconcentração do Terciário de Gestão na Cidade de São Paulo. Dissertação de Mestrado. São Paulo: Universidade de São Paulo, FFLCH, Departamento de Geografia.

Gaeta, A. C. (1995). São Paulo: fisionomia urbana e fetiche. Tese de Doutorado. São Paulo: Universidade de São Paulo, FFLCH, Departamento de Geografia.

Goe, R.W. (1996). "An examination of relationship between corporate spatial organization, restructuring, and external contracting of producer services within a metropolitan region". Urban Affairs, 32, 1, 23-45.

Gottdiener, M. (1993). A produção social do espaço urbano. São Paulo: Edusp.

Harvey, D. (1980). A Justiça Social e a Cidade. São Paulo: Hucitec.

Hepworth, M. E. (1989). Geography of the information economy. Londres: Belhaven Press.

Lefebvre, H. (1976). Espacio y politica: el derecho a la ciudad II. Barcelona: Ediciones Península.

Lencione, S. (2003). "Cisão territorial da indústria e integração regional no estado de São Paulo”. En: M. F. Gonçalves et al. (orgs.). Regiōes e cidades, cidades nas regiōes: o desafio urbano-regional. São Paulo: Ed. UNESP / ANPUR.

Malezieux, J. (1993). "L'immobilier d'entreprise: dynamique spatiale". En M. Savy y P. Veltz (dirs.) Les nouveaux espaces de l'entreprise. Paris: Datar/Editions de l'Aube.

Malezieux, J. (1996). "La production de l'espace urbain et ses acteurs - Immobilier d'entreprise et spatiale". Mimeo, 22.

Marshall, J. N.. (1994). "Business reorganization and the development of corporate services in metropolitan areas". The Geographical Jornal, 160, 1, 41-49.

Ribeiro, A. C. T. (1996). Urbanidade e Vida Metropolitana. Rio de Janeiro: Jobran Editora.

Sánchez, J. E. (1991). Espacio, economía y sociedad. Madrid: Siglo XXI.

Santos, M. (1977). "Society and space. Social formation as theory and method". Antipode, 9, 1, 3-13. 
(1990). Metrópole Corporativa Fragmentada. São Paulo: Nobel.

(1994a). Por uma economia política da cidade. São Paulo: Hucitec.

(1994b). Técnica Espaço Tempo: globalização e meio técnico-científicoinformacional. São Paulo: HUCITEC.

(1995). "Raison universelle, raison locale.

Les espaces de la rationalité", Espaces et Societé, 79, 108-110.

(1996). A natureza do espaço: técnica e tempo. Razão e emoção. São Paulo: Hucitec. (2000). Por uma outra globalização: do pensamento único à consciência universal. Rio de Janeiro: Record.

Sassen, S. (1991). The global city: New York, London, Tokyo. Princeton: University Press.

Silva, A. M. B. (2004). "A metrópole de São Paulo e as empresas produtoras de informação: as firmas de consultoria”. Revista Estudios Socioterritoriales, 4. Tandil, Universidad Nacional del Centro de la Provincia de Buenos Aires. (2003). "O processo de mundialização da metrópole de São Paulo: tendências e conflitos". En: M. A. Souza (org.) Território Brasileiro. Usos e Abusos. Campinas: Ediçōes Territorial.

Veltz, P. (1997). Mondialisation, villes et territoires. Léconomie d'archipel. Paris: Presses Universitaires de France.

Weeler, J. O. \& Mitchelson, R. J. (1989). "Information flows among major metropolitan areas in the United States". Annals of the Association of American Geographers. 79 (4)

Wood, P. A. (1996). "Business services, the management of change and regional development in the UK". Transaction of the Institute of British Geographers, 21 (4), 644-665. 